\title{
Rate Optimization for IDMA Systems with Iterative Joint Multi-User Decoding
}

\author{
Tao Yang, Student Member, IEEE, Jinhong Yuan, Member, IEEE, and Zhenning Shi, Member, IEEE
}

\begin{abstract}
In this letter, we develop a rate allocation scheme for interleave-division multiple-access (IDMA) systems with iterative decoding. We use a fully-analytical approach to predict the performance of the scheme. Then, we propose a modified linear programming method to find the best rate profile for the scheme. Numerical results show that with just repetition coding and optimal rate allocation, the performance of the scheme is only about $5 \mathrm{~dB}$ away from the capacity for a wide range of SNR, provided that the number of users is sufficiently large. Compared with power allocation schemes for IDMA, the proposed rate allocation scheme achieves a similar performance at a moderate spectral efficiency and the requirement of sophisticated power amplifiers can be relaxed.
\end{abstract}

Index Terms-Multi-user detection, iterative decoding, CDMA, power allocation.

\section{INTRODUCTION}

$\mathbf{F}$ ROM information theory, it is well-known that the maximum sum-capacity of a multiple-access channel (MAC) can be achieved by using successive cancellation (SC) together with power allocation (PA), provided that capacity-achieving error control codes are employed [1]. Unfortunately, an ideal code does not exist and the SC-PA technique, which is also referred to as "onion-peeling (OP)", is subjected to excessive power consumption. For a system with poor codes, the extra power consumption could be so significant that the performance of the system with onion-peeling is far from being acceptable [2].

Nowadays, it has been widely understood that the total power can be reduced if an iterative "soft onion-peeling (SOP)" technique [3] is employed. In this approach, the multiple-access interference is gradually removed [4] rather than being successively and completely cancelled, typically from the strongest user to the weakest user. If the power distribution is designed properly, the system with SOP can approach the interference-free performance after a number of iterations.

Recently, it has been shown that for a system with equal power distribution, the capacity bound can also be achieved

Manuscript received April 1, 2008; revised July 30, 2008, October 9, 2008, and November 18, 2008; accepted November 22, 2008. The associate editor coordinating the review of this paper and approving it for publication was $\mathrm{H}$. Nguyen.

T. Yang and J. Yuan are with the School of Electrical Engineering and Telecommunications, University of New South Wales, Sydney, N.S.W. 2052, Australia (e-mail: t.yang@student.unsw.edu.au, j.yuan@unsw.edu.au).This work was supported by the Australian Research Council (ARC) and was in a part of ARC discovery project DP0667030.

Z. Shi is with National ICT Australia and affiliated with the Australian National University (e-mail: zhenning.shi@nicta.com.au). National ICT Australia is funded through the Australian Government's backing Australia's Ability initiative and in part through the Australia Research Council.

Digital Object Identifier 10.1109/TWC.2009.080438 by adopting unequal rate allocation (RA) and successive cancellation, as long as the number of user rate groups is sufficiently large [5]. In a system with iterative SOP and unequal rate distribution, low-rate users converge faster than high-rate users and the operation of the iterative receiver is facilitated by using the rate allocation.

So far, we have seen that the iterative decoding (ID) of multi-user systems can be enhanced by either power allocation or rate allocation. In literature, the PA method for iterative SOP systems, such as interleave-division multiple-access (IDMA) systems [6][7], has been intensively investigated. To the best of our knowledge, the research on the rate allocation for the iterative SOP systems is rather limited. As a matter of fact, there are some inherent advantages of rate allocation over power allocation in practical applications, e.g. the requirement of the sophisticated power amplifier can be relaxed.

In this letter, we study the rate allocation for equal-power IDMA systems in AWGN channels. We develop a fullyanalytical rate allocation approach for an IDMA system with repetition coding. The performance of the systems can be precisely predicted via a variance evolution technique. For a wide range of signal-to-noise ratio, we observe that with just repetition coding and rate allocation, the performance is only about $5 \mathrm{~dB}$ away from the capacity limit at a bit error rate (BER) of $10^{-4}$, provided that the number of users is sufficiently large. Moreover, the achievable rate is almost parallel to the Shannon-bound. Compared with the power allocation scheme for IDMA, the proposed rate allocation scheme achieves a similar performance at a moderate spectral efficiency, whereas it suffers a fairly small power loss at a high spectral efficiency.

The letter is structured as follows. Section II reviews an IDMA system with iterative decoding. Section III introduces the variance evolution technique which is employed to predict the performance of the IDMA systems. In particular, we provide an upper bound on the code variance transfer characteristics of repetition codes such that the performance of a repetition-coded IDMA system is fully-analytical. In section IV, we depict the rate allocation technique for the IDMA system with equal power distribution. The optimal rate allocation factors are obtained by employing an modified linear programming (LP) method. In section V, the numerical results and performance bounds are given. Finally, we draw the conclusion in section VI.

\section{IDMA SYSTEM MODEL}

The block diagram of a generic IDMA system is depicted in Fig. 1. In this system, the $k$ th user's information $b_{k}$ is encoded by a channel code, yielding coded sequence $d_{k}$. Then, 


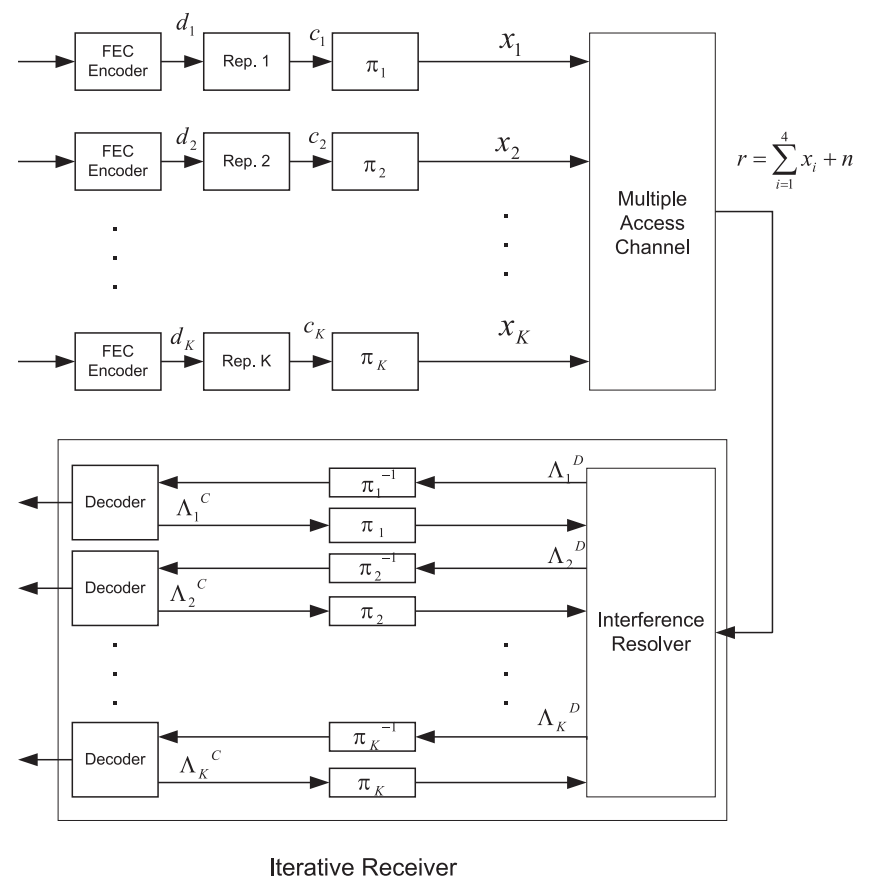

Fig. 1. Architecture of an IDMA transceiver for 4 users.

$d_{k}$ is encoded by a repetition code with length $L_{k}$, yielding discrete chips $c_{k}(t), t=1,2, \ldots, T$ where $T$ is the number of chips for each decoding frame and $t$ indexes the $t$ th chip of each frame. Note that $T$ is also the interleaver size of the IDMA system. Then, the chips are interleaved by $\pi_{k}$ and BPSK modulated, yielding chip-interleaved symbol sequence $\mathbf{x}_{k}=\left[x_{k}(1), x_{k}(2), \ldots, x_{k}(T)\right]$. Note that the rates of the repetition codes for different users are not the same. The chipinterleaved signals go through a multiple-access channel and the received signal is modelled as

$$
r(t)=\sum_{k=1}^{K} x_{k}(t)+n(t)
$$

which is the superposition of the chip-interleaved signals from all users plus the noise. In (1), $n(t)$ is an AWGN with variance $\sigma_{n}^{2}$. The receiver at the base station is required to decode all users' information which are distinguished by their specific interleavers.

Now, let us have a brief review of the iterative decoding of this IDMA model. We consider an equal-power system in which each chip has a unit power. In each iteration, the interference resolver (multi-user detector) first computes the chip-level estimates in the form of log-likelihood ratios (LLRs), given by

$$
y_{k}(t)=2 \frac{r(t)-E\left[\zeta_{k}(t)\right]}{\operatorname{Var}\left[\zeta_{k}(t)\right]} .
$$

where

$$
E\left[\zeta_{k}(t)\right]=\sum_{k^{\prime}=1, k^{\prime} \neq k}^{K} E\left[x_{k^{\prime}}(t)\right]
$$

and

$$
\operatorname{Var}\left[\zeta_{k}(t)\right]=\sum_{k^{\prime}=1, k^{\prime} \neq k}^{K} \operatorname{Var}\left[x_{k^{\prime}}(t)\right]+\sigma_{n}^{2}
$$

are the estimated statistical mean and variance of the interference plus noise. After chip-level de-interleaving, the chip LLRs w.r.t a specific coded bit are summed to yield the bitlevel soft information. That is,

$$
\Lambda^{D}\left[\left(d_{k}(i)\right]=\sum_{j=1}^{L_{k}} y_{k}\left[(i-1) \times L_{k}+j\right)\right]
$$

where $\Lambda^{D}\left[\left(d_{k}(i)\right]\right.$ stands for the bit-level LLR obtained from the detector. The bit-level LLRs are forwarded to the FEC decoders which employ maximum a posteriori probability (MAP) decoding to update the statistical mean of the coded bits as well as the chip-level statistics $E\left[x_{k}(t)\right]$ and $\operatorname{Var}\left[x_{k}(t)\right]$. Subsequently, the most recent chip-level statistics $E\left[x_{k}(t)\right]$ and $\operatorname{Var}\left[x_{k}(t)\right]$ are feedback to the detector. In the next iteration, by using a better statistics, the detector generates refined chip-level LLRs. For more details about the iterative decoding for IDMA, please refer to [6][7].

The virtue of IDMA system is that for each transmitted symbol, the interference for one chip is independent from that for another chip due to the chip-interleaver. Hence, the optimal filtering process after the interference cancellation is simply the summation of the log-likelihood-ratio (LLR) values for all the chips, enabling a low-complexity but yet powerful detector for the system.

\section{Performance Analysis of IDMA Systems}

In this section, we first provide a variance transfer function in a closed form for a repetition code. Then, we illustrate the variance evolution for the performance analysis of the IDMA system.

\section{A. Variance Transfer Function of Repetition Codes}

Consider a repetition-coded IDMA system with $K$ users. As illustrated in section II, the $k$ th user is encoded by a repetition code with length $L_{k}$, where $L_{k} \in\left[L_{\min }, L_{\max }\right]$. For simplicity, we only consider the case that $L_{k}$ is an integer. The signal to noise ratio (SNR) is $\rho=K / \sigma_{n}^{2}$ where we assume unit chippower for each user.

We define $\sigma_{k}^{2}$ the variance of interference plus noise at the detector's output for the $k$ th user. Let $E\left[1-\tanh \left(\Lambda^{C}\left(d_{k}\right)\right)\right]^{2}$ be the bit-variance [9] of the output signal from user- $k$ 's repetition-code decoder, where $\Lambda^{C}\left(d_{k}\right)$ is the bit-level LLR from the repetition-code decoder. Note that for a repetition decoder, the output bit-variance is a function of the input variance $\sigma_{k}^{2}$ as well as the length of the repetition code. This function is referred to as a variance transfer (VT) function [9]. Now, we present the following lemma.

Lemma 1: Let $\sigma_{k}^{2}$ denote the input variance to the $k$ th user's repetition code decoder whose repetition length is $L_{k}$. The variance transfer function of the repetition code for the $k$ th user is upper-bounded by [5][8]

$$
f\left(\sigma_{k}^{2}, L_{k}\right) \leq\left\{\begin{array}{c}
\frac{1}{1+\frac{L_{k}-1}{\sigma_{k}^{2}}}, \text { if } \sqrt{\frac{L_{k}-1}{\sigma_{k}^{2}}}<1 \\
\pi Q\left(\sqrt{\frac{L_{k}-1}{\sigma_{k}^{2}}}\right), \text { otherwise }
\end{array}\right.
$$




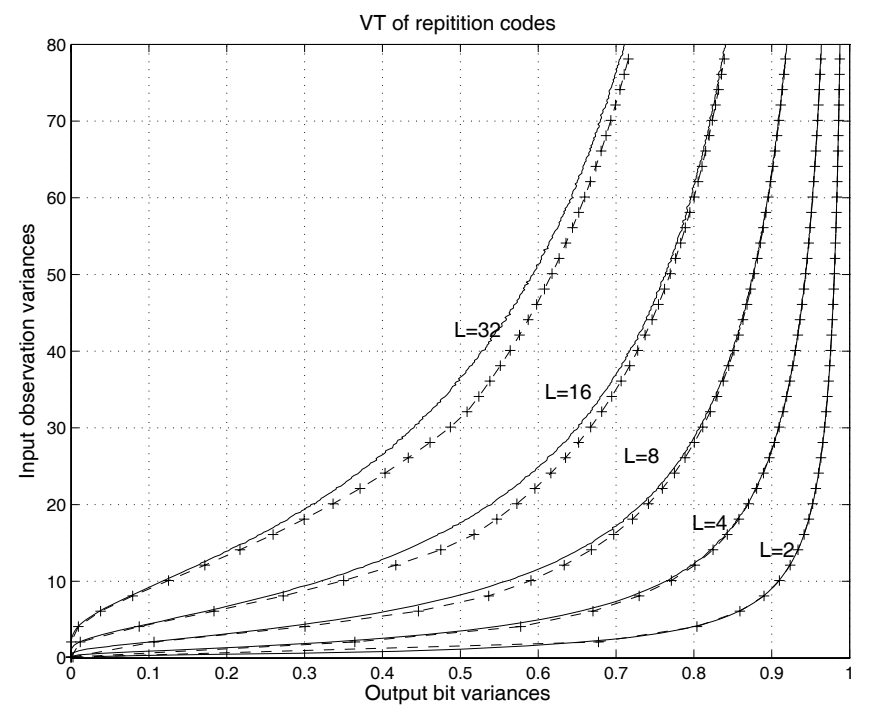

Fig. 2. Variance transfer function of repetition codes. The solid curves are the genuine VT obtained from simulation. The dashed curves with "+" are from the closed-form given in (6).

where $f\left(\sigma_{k}^{2}, L_{k}\right)$ is the output bit-variance from the $k$ th decoder for repetition code and $Q(x)$ is the Gaussian error function [5].

The VT function curves for repetition codes with various lengths are shown in Fig. 2. The vertical axis denotes the input variances of interference plus noise and the horizontal axis stands for the output bit variances. From this figure, it is clear that (6) is very accurate for repetition codes with small lengths. However, as the code length increases, the difference between (6) and the genuine VT function becomes larger, especially for the region that $\sqrt{\frac{L_{k}-1}{\sigma_{k}^{2}}}$ is close to 1 . Therefore, the performance analysis by using (6) provides an upper bound on the BER (or a lower bound on the achievable sum rate). Later on, we will see that good results can be yielded by using (6).

\section{B. Variance Evolution}

Now, we illustrate the variance evolution technique. For two consecutive iterations, the variance of the $k$ th user at the detector's output is updated as follows

$$
\sigma_{k, q}^{2}=\underbrace{\sum_{k^{\prime}=1}^{K} f\left(\sigma_{k^{\prime}, q-1}^{2}, L_{k^{\prime}}\right)-f\left(\sigma_{k, q-1}^{2}, L_{k}\right)}_{\text {residual interference }}+\sigma_{n}^{2} .
$$

where " $q$ " is the index for the $q$ th iteration. As the receiver iterates, the variance $\sigma_{k, q}^{2}, k=1,2, \ldots, K$ is gradually reduced. After a number of iterations, the residual interference becomes small if the iterative decoding process converges. At the final iteration, we have

$$
B E R_{k}^{\infty}=\frac{1}{2} \operatorname{erfc}\left(\sqrt{\frac{1}{2 \sigma_{k, \infty}^{2}}}\right)
$$

which is the predicted bit error ratio (BER) for the user- $k$ at the final iteration. The total BER with respect to all the users is given by

$$
B E R^{\infty}=\frac{\sum_{k} \frac{B E R_{k}^{\infty}}{L_{k}}}{\sum_{k} \frac{1}{L_{k}}} .
$$

Later, we will see that this simple variance evolution approach is able to give a good prediction of the BERs of the unequalrate IDMA systems.

\section{RATE AlLOCATION FOR EQUAL-POWER IDMA SYSTEMS}

In this section, we illustrate the rate allocation for the repetition-coded IDMA systems with equal power distribution. The advantage of using non-identical rate for different user is that the strong user (low-rate user) can converge earlier than the weak user (high-rate user) and thus the convergence of iterative decoding is facilitated.

In the $q$ th iteration, the residual interference plus noise, after interference cancellation (IC) [11] and with respect to the $k$ th user, is given in (7). A sufficient condition for the iterative receiver to converge, i.e. the overall BER is very small, is

$\sigma_{k, q}^{2}=\sum_{k^{\prime}=1}^{K} f\left(\sigma_{k^{\prime}, q-1}^{2}, L_{k^{\prime}}\right)-f\left(\sigma_{k, q-1}^{2}, L_{k}\right)+\sigma_{n}^{2} \leq(1-\delta) \sigma_{k, q-1}^{2}$

for all user-index $k$ and for the variance range $\sigma_{k, q-1}^{2} \in$ $\left[\sigma_{\min }^{2}, \sigma_{\max }^{2}\right]$, where $\sigma_{\max }^{2}=K-1+\sigma_{n}^{2}$ and $\sigma_{\min }^{2}$ is determined by the SNR and the target BER. The parameter $\delta$ in (10) is of a small value which affects the convergence speed. Given the above condition, the variance of each user keeps reducing throughout the whole iterative process and the iterative receiver converges. The details for the convergence behavior analysis of an iterative receiver can be found in [5][9].

If the number of users $K$ becomes very large, i.e. $K \rightarrow \infty$, we can assume that the Lindeberg condition [5] is satisfied. Here, the Lindeberg condition means that as the number of users goes to infinity, the ratio between the variance of any user and the total variance of all users approaches zero, that is

$$
\lim _{K \rightarrow \infty} \frac{\sigma_{k^{\prime}}^{2}}{\sum_{k=1}^{K} \sigma_{k}^{2}}=0, k^{\prime}=1,2, \ldots, K
$$

In practice, we found that for $K \geq 24$, the variance of any user is very tiny compared to the total variance of all users. As a result, the variance of the iterative process become independent of the user index and the variance for all users approaches the value

$$
\sigma_{q}^{2}=\sum_{k=1}^{K} f\left(\sigma_{q-1}^{2}, L_{k}\right)+\sigma_{n}^{2}
$$

Then, (10) becomes

$$
\sum_{k=1}^{K} f\left(\sigma_{q}^{2}, L_{k}\right)+\sigma_{n}^{2} \leq(1-\delta) \sigma_{q}^{2}, \sigma_{q}^{2} \in\left[\sigma_{\min }^{2}, \sigma_{\max }^{2}\right] .
$$

where $\sigma_{q}^{2}$ is the total variance of all the users and the additive noise. The above observation indicates that for a large system 
case, it is allowed that the system behavior can be described by a single-parameter dynamical system, similar to [9][10]. In the optimization, (12) becomes the constraints. Therefore, the RA problem becomes finding $\max \sum_{k} \frac{1}{L_{k}}$ subject to (12).

Let $M=L_{\max }-L_{\min }+1$ be the total number of possible lengths of the repetition codes and $L(m)$ be the code-length of the $m$ th repetition code. Note that in order to ensure that the weakest user can achieve the target SNR, we have

$$
L_{\min }>2\left[\operatorname{erfc}^{-1}\left(2 \cdot B E R_{\text {target }}\right)\right]^{2} \cdot \sigma_{n}^{2} .
$$

Let $m \in[1, M]$ and $N(m)$ be the number of users assigned to a rate of $1 / L(m)$. Then, the constraints become

$$
\begin{gathered}
\sum_{m} N(m) f\left[\sigma^{2}, L(m)\right]+\sigma_{n}^{2} \leq(1-\delta) \sigma^{2}, \sigma^{2} \in\left[\sigma_{\min }^{2}, \sigma_{\max }^{2}\right] \\
\sum_{m} N(m)=K
\end{gathered}
$$

and our objective function becomes $\max \sum_{m} \frac{N(m)}{L(m)}$. Please note that the iteration index " $q$ " is omitted in the above constraint.

Generally, the best rate profile $N(m), m=1, \ldots, M$ can be found via two methods. The most straightforward method is to exhaustively search the best rate profile. However, this approach is of a prohibitive complexity even for a moderate number of users. The second approach is to use linear programming (LP) since constraints and the objective function are linear.

Typically, the solutions of $N(m)$ from the LP are not integers. For a large system, intuitively, we may consider that the ratio between the non-integer solution $N(m)$ and the total number of users $K$ indicates the "portion of users" allocated to a certain rate. However, in practice, the integersolutions are almost exclusively of interests. Therefore, the best rate distribution should be computed by using "integer programming", where the constraints remain the same as (14) but $N(m)$ are within the set of positive integers.

In our work, we consider two cases. Firstly, we consider a "large-system" scenario that there is no constraints on the number of users. For this case, we will see a lower bound on the achievable rate predicted by the variance evolution. In the second place, we consider a "small-system" scenario in which we have limited number of users. In this letter, we regard a system with less than 100 users as a "small-system". Usually, integer programming can be realized by using branching-andbounding algorithm on top of linear programming to get the integer solutions. Unfortunately, the number of sub-problems may become very huge even for a moderate number of users. Therefore, we make some simple modifications on LP to yield the integer solutions.

1) Case 1 Large systems: Suppose that the rate allocation operation was carried out based on $K$ users and we obtained the rate profile $N(m), m=1, \ldots, M$ which are not integers for the base system. If there is no constraints on the number of users, we may enlarge the base system by a factor of $F$, resulting an $F \cdot K$-user system. While enlarging the system, we decrease the rate of each repetition code by a factor of $1 / F$ so that the sum-rate is maintained. Consequently, the rate profile becomes $N^{\prime}(m)=F \cdot N(m), L^{\prime}(m)=$ $F \cdot L(m), m=1, \ldots, M$. After that, we round $F \cdot N(m)$ to its closest integer, yielding $\bar{N}(m), m=1, \ldots, M$ which is the new rate distribution for this system with $F \cdot K$ users. As long as $F$ is large enough, the rounding error is negligible.

In fact, this approximation by enlarging the system is not strictly the optimal rate profile for an $F \cdot K$-user system. However, by using the variance evolution for this $F \cdot K$ user system with the rate profile $N^{\prime}(m)=F \cdot N(m)$, $L^{\prime}(m)=F \cdot L(m), m=1, \ldots, M$., we find that the enlarged system can always converge to a very small variance. The reason for this behavior is that, under the same condition, i.e., with the same a priori information from the decoders, the bitlevel SINR of the enlarged system is larger than that of the base system. More details are given as follows:

Let $\rho_{B}$ and $\rho_{E}$ denote the bit-level SINRs of the base system and the enlarged system, respectively. By enlarging the system, while reducing the code rate of each user by a factor of $1 / F$, we may regard that there are $F$ times more chips associated with each bit. Let us consider that the same a priori information for $m=1, \ldots, M$ is fed to the detector of the base system and to that of the enlarged system. Then, in the enlarged system, since the number of users is enlarged by $F$, the total variance of interference is also enlarged by $F$ and the variance of interference plus noise is enlarged by a factor of $G$ which is less than $F$. Consequently, the chip-level SINR of the enlarged system is reduced by a factor of $1 / G$. However, since there are $F$ times more chips associated with one bit, after the chip-LLR combining given in (5), we have

$$
\rho_{E}=\frac{F}{G} \rho_{B}>\rho_{B} .
$$

Since the bit-level SINR dictates the decoder's output variance [5] and we have (15), the decoder can yield a smaller output variance in the enlarged system, as compared to that of the base system. Therefore, the performance of the enlarged system is not worse than the base system.

For a large system with $F \cdot K$ users, using the Monte Carlo simulation to find the BER is time-consuming. Therefore, we will just use variance evolution to find an upper bound on the BER and to obtain a lower bound on the achievable sumrate. In the next section, we will show the sum-rate for this large-system scenario.

2) Case 2 Small systems: Now, we consider a small-system scenario. Please note that "small-system" refers to the system which cannot be "enlarged". Again, let us suppose that the rate allocation operation was carried out based on $K$ users and we obtained the rate profile $N(m), m=1, \ldots, M$ which are not integers. If it is not allowed to enlarge the system, as is described earlier, we should round the non-integers to integers in a smart way.

In our work, we first round (towards the lower floor) the rate allocation factors to the nearest integers, resulting a new rate distribution. Let $\lfloor\cdot\rfloor$ denotes the operation of rounding towards the lower floor. For example, if $N(m)=8.5$, after the rounding, we have $N^{\prime}(m)=\lfloor N(m)\rfloor=8$. If we take $N^{\prime}(m)=\lfloor N(m)\rfloor, m=1, \ldots, M$ as the new rate profile, the aggregated number $\sum_{m} N^{\prime}(m)$ is obviously smaller than total number of users $K$. Normally, $K-\sum_{m} N^{\prime}(m)$ is of value 1 


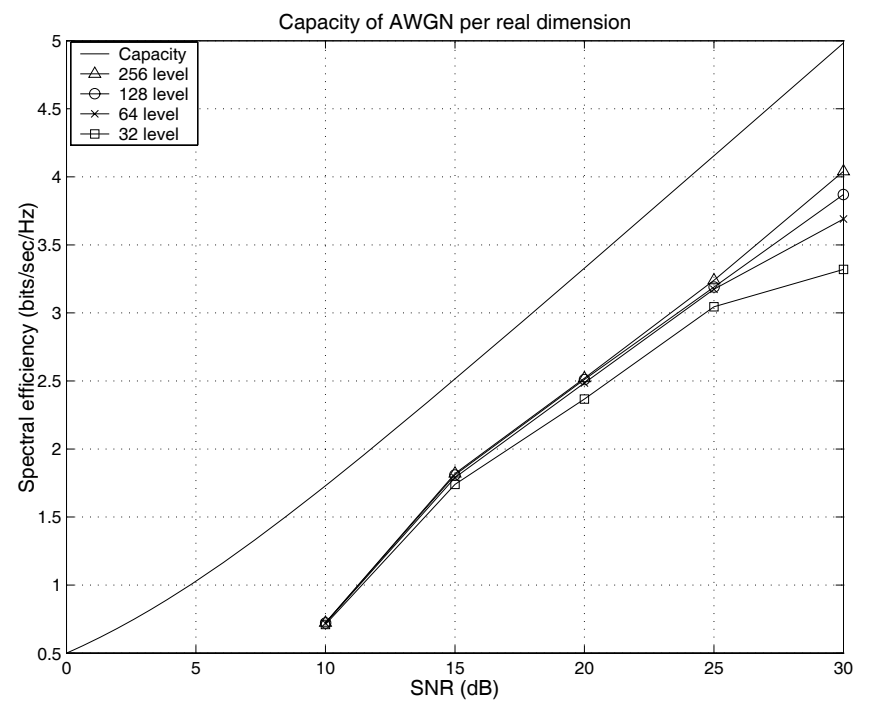

Fig. 3. Achievable sum-rate of the unequal-rate IDMA systems for each real dimension and for $\mathrm{BER}<10^{-4}$.

or 2. At this stage, there are still one or two users whose code rates have not been allocated yet. We only need to find the best code rates for these (one or two) users. This can be performed by simply searching for the best rates which, together with $N^{\prime}(m)$, satisfy the constraints. In such a way, the integer-solution for this scenario is obtained. Although it is not possible to prove that this solution is precisely the optimal integer solution, we will see in the next section that the solution works very well in both the variance evolution and Monte Carlo simulations. At the same time, this approach is much easier than the integer-programming.

\section{NumericAl RESUltS}

In this section, we present the numerical results for the unequal-rate, equal-power and repetition-coded IDMA systems.

\section{A. Case 1 Large systems}

For the large-system case, we use variance evolution to predict the lower bound on the achievable sum rate of the systems, which is depicted in Fig. 3. The Shannon-capacity bound is also included in the figure. To find the results in Fig. 3, we first perform linear programming based on $K=32$ $(64,128,256)$ levels and we obtain the non-integer solutions. Then, we enlarge the system by a factor of $F$, i.e. $F=5$, resulting an enlarged $160(320,640,1280)$-user system. After that, variance evolution is used to predict the performance and the achievable sum-rate of this enlarged system.

From Fig. 3, we observe that the rate allocation based on a larger value of $K$ always yields better performance and the advantage becomes more significant as the SNR goes up. Moreover, we see that the achievable sum-rate for a system with a reasonably large number of users is parallel to the Shannon-capacity and the difference is around $5 \mathrm{~dB}$ for a wide range of SNR.

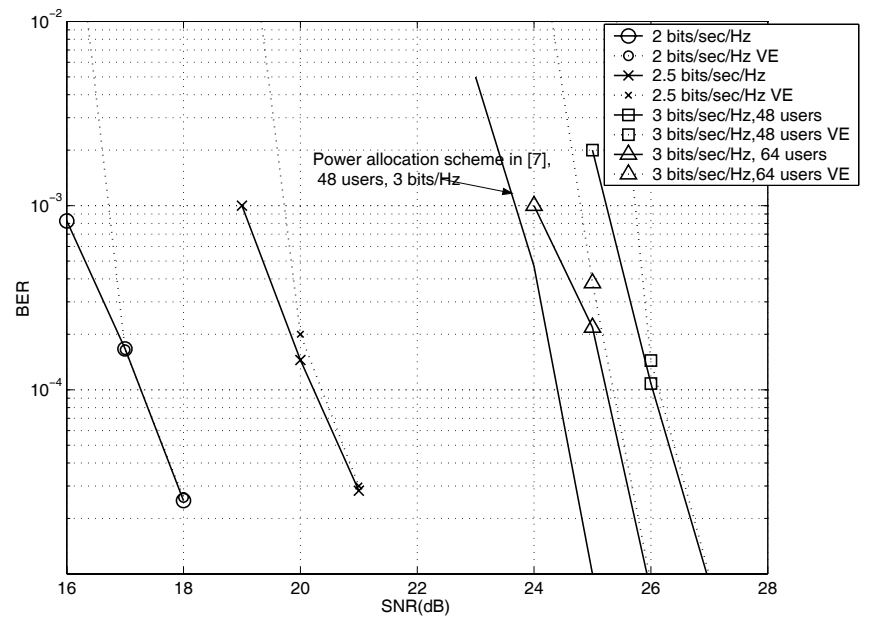

Fig. 4. BER performance of unequal-rate IDMA systems. In all the simulations and variance evolution, we simply use sufficiently large numbers of iterations to yield the results.

\section{B. Case 2 Small systems}

For the small system case, we do not enlarge the system and we only consider $K=32(40,48,64)$ users. After the linear programming, the non-integers are properly rounded to integers, as mentioned in section IV. 2).

In Fig. 4, we plot the BER performance of the small systems evaluated by computer simulation, where the rate profile is specified in Table. 1. The predicted bit error ratio by using variance evolution is also included. For a fair comparison with the power allocation scheme in [7], we use the same number of users as in [7] to achieve a certain spectral efficiency ${ }^{1}$. From Fig. 4, we see that the system with RA converges very well and the "water-fall" region in the BER curve is apparent. At a small BER region, the predicted bit error ratio from variance evolution is very close to the simulated BER. We also include a scheme where a spectral efficiency of $3 \mathrm{bits} / \mathrm{sec} / \mathrm{Hz}$ is achieved with 64 users. By using more users for rate allocation, a noticeable performance improvement is achieved.

Now, we compare the RA schemes with the power allocation schemes in [7]. At a a spectral efficiency of 2 bits $/ \mathrm{sec} / \mathrm{Hz}$, the RA scheme based on 32 users achieves almost the same BER performance as the PA scheme in [7], which is not depicted in Fig. 4. At 3 bits/sec/Hz, the RA scheme based on 48 users is about $2 \mathrm{~dB}$ worse than the PA scheme in [7]. However, if we use a larger number of users, i.e. 64 users, to achieve that spectral efficiency, the gap is significantly reduced. Note that in the RA scheme, we only use integer rates which can be easily implemented by digital circuits. However, in [7], the resolution of the quantized power levels could be arbitrarily small, thus the design of power amplifier could be very demanding. The performance of the RA scheme may be further improved if the rate optimization is carried out over non-integer rates which is not discussed in this letter.

In Fig. 5, we plot the BERs of the iterative receiver for different number of iterations, where the SNR is fixed to 26 $\mathrm{dB}$. In the figure, we compare the PA scheme in [7] with 48

\footnotetext{
${ }^{1}$ In [7], 2 bits/sec/Hz is achieved with 32 users and 3 bits/sec/Hz is achieved with 48 users.
} 


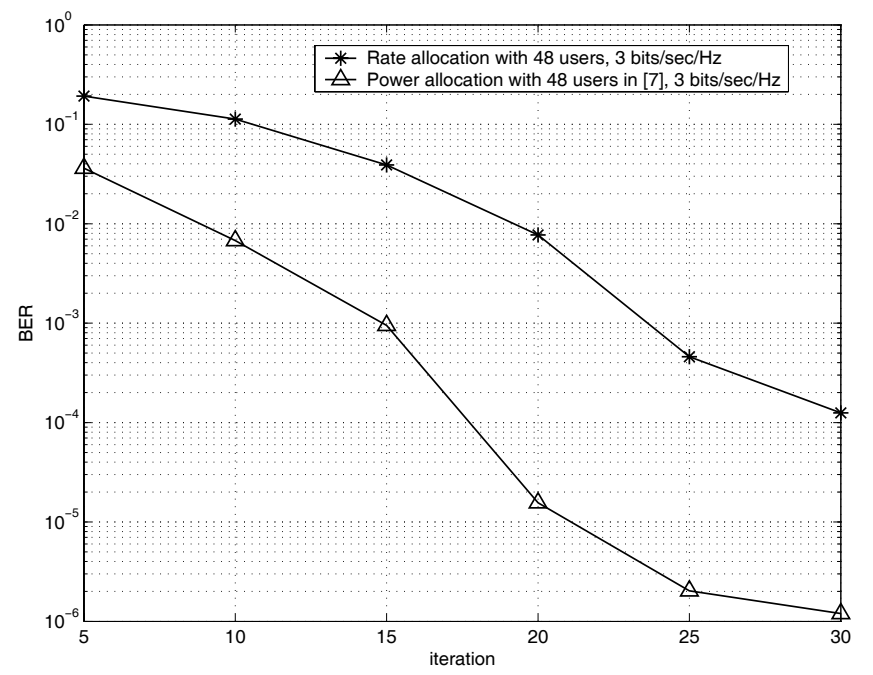

Fig. 5. BER performance with different iterations.

TABLE I

RATE ALLOCATION

\begin{tabular}{|l|l|}
\hline & Length $\times$ no. of users \\
\hline$K=32,(2 \mathrm{bits} / \mathrm{sec} / \mathrm{Hz} / \mathrm{dim})$ & $8 \times 11,12 \times 1,37 \times 20$ \\
\hline$K=40,(2.5 \mathrm{bits} / \mathrm{sec} / \mathrm{Hz} / \mathrm{dim})$ & $5 \times 6,7 \times 1,28 \times 4,29 \times 29$ \\
\hline$K=48,(3 \mathrm{bits} / \mathrm{sec} / \mathrm{Hz} / \mathrm{dim})$ & $2 \times 2,3 \times 1,22 \times 26,24 \times 1,46 \times 18$ \\
\hline$K=64,(3 \mathrm{bits} / \mathrm{sec} / \mathrm{Hz} / \mathrm{dim})$ & $3 \times 4,7 \times 1,31 \times 5,32 \times 33,62 \times 21$ \\
\hline
\end{tabular}

users ( $3 \mathrm{bits} / \mathrm{sec} / \mathrm{Hz})$ and the proposed rate allocation scheme with 48 users ( 3 bits/sec/Hz) which is specified in Table I. We see that both schemes requires a large number of iterations to converge. This is phenomenon typical for schemes with high spectral efficiency. Moreover, the RA scheme converges slightly slower than the PA scheme.

\section{CONCLUSION}

In this correspondence, we developed the rate allocation for IDMA systems with iterative detection and decoding in AWGN channel. We used a fully-analytical approach to predict the performance of the scheme. Then, we proposed a modified LP method to find the rate profile for the scheme. Numerical results shows that with just repetition coding and
RA, the scheme is only about $5 \mathrm{~dB}$ away from the capacity for a wide range of SNR, provided the number of users is large enough. Compared with the power allocation scheme in [7], the proposed RA scheme suffers a small power loss at a high spectral efficiency. However, the implementation of the RA scheme becomes easier since the requirement of the sophisticated power amplifier can be relaxed.

For general fading channels, the rate would be adaptive to the received power, which is not uniform in a near-far scenario. Therefore, the proposed scheme needs to monitor the received power of all active users [7] to perform rate optimization and this will be investigated in future works.

\section{REFERENCES}

[1] T. M. Cover and J. A. Thomas, Elements of Information Theory, 2nd ed. Wiley, 2006.

[2] X. Ma and P. Li, "Power allocation for multilevel coding with sigma mapping," Electron. Lett., vol. 40, no. 10, May 2004.

[3] N. Chayat and S. Shamai, "Iterative soft onion peeling for multi-access and broadcast channels," in Proc. IEEE PIMRC, vol. 3, pp. 1385-1390, Sept. 1998.

[4] X. Wang and H. V. Poor, "Iterative (turbo) soft interference cancellation and decoding for coded CDMA," IEEE Trans. Commun., vol. 47, pp. 1046-1061, July 1999.

[5] C. Schlegel, Z. Shi, and M. Burnashev "Optimal power/rate allocation and code selection for iterative joint detection of coded random CDMA," IEEE Trans. Inform. Theory, vol. 52, no. 9, Sept. 2006.

[6] P. Li, L. Liu, K. Wu, and W. K. Leung, "Interleave-division multipleaccess," IEEE Trans. Wireless Commun., vol. 5, no. 4, pp. 938-947, Apr. 2006.

[7] L. Liu, T. Jun, and P. Li, "Analysis and optimization of CDMA systems with chip-level interleavers," IEEE J. Select. Areas Commun., vol. 24, no. 1, pp. 141-150, Jan. 2006.

[8] M. V. Burnashev, C. Schlegel, W. A. Krzymien, and Z. Shi, "Mathematical analysis of one successive cancellation scheme in iterative decoding," Probl. Inform. Trans., vol. 40, no. 4, Jan. 2004.

[9] Z. Shi and C. Schlegel, "Iterative multisuser detection and error control code decoding in random CDMA," IEEE Trans. Signal Processing, vol. 54, no. 5, pp. 1886-1895, May 2006.

[10] P. Alexander, A. Grant, and M. C. Reed, "Iterative detection in codedivision multiple-access with error control coding," European Trans. Telecommun., vol. 9, Special Issue on CDMA Techniques for Wireless Communications Systems, pp. 419-426, Sept./Oct. 1998.

[11] T. Yang, J. Yuan, and Z. Shi, "Jointly Gaussian approximation and multi-stage LLR combining in the iterative receiver for MIMO-BICM systems," IEEE Trans. Wireless Commun., vol. 7, no. 12, pp. 5250-5256, Dec. 2008. 